\title{
Evidências de Validade da Bateria de Provas de Raciocínio (BPR-5) para Seleção de Pessoal
}

\author{
Evidences on the Validity of the Battery of Reasoning Tests (BPR-5) \\ for Employment Selection
}

\author{
Viviane de Oliveira Baumgartl ${ }^{a}$ * \& Ricardo Primi ${ }^{b}$ \\ Pontifícia Universidade Católica de Minas Gerais, Belo Horizonte, Brasil a , Universidade São Francisco, Itatiba, Brasil ${ }^{b}$
}

\begin{abstract}
Resumo
A utilização de testes psicológicos é uma prática comum em empresas brasileiras. Estas avaliações, no entanto, muitas vezes são realizadas sem levar em conta a eficácia dos instrumentos utilizados em discriminar critérios relevantes para um bom de desempenho do funcionário em seu posto de trabalho. Nesse sentido o presente estudo teve como objetivo verificar as evidências de validade do BPR-5 em um contexto organizacional. Os participantes foram 79 funcionários de uma empresa de energia elétrica brasileira. Como critério foi utilizado o número de acidentes de trabalho dos funcionários. Os dados foram analisados por meio de estatísticas descritivas e análises correlacionais. Nos resultados, o critério utilizado, referente ao número de acidentes de trabalho, correlacionou-se com a inteligência principalmente para funcionários com menor tempo na função ($0,39 ; \mathrm{p}<0,05)$. As implicações dos resultados tanto para a pesquisa quanto para a prática são discutidas.

Palavras-chave. Seleção de pessoal; validade; inteligência; setor elétrico.
\end{abstract}

\begin{abstract}
The use of psychological tests is a common practice among Brazilian companies. These evaluations, however, are done many times without taking into account the efficiency of the instruments that are used for discriminating relevant criteria for a good performance of the employee at work. The purpose of this study was to check evidences on the validity of the BPR-5 test in an organizational context. The sample consisted of 79 employees of a Brazilian electric company. The number of injuries at the workplace was used as criteria. The data was analyzed using descriptive statistics and correlation analyses. The results showed that the criteria of injuries at the workplace presented significant correlations with the tests, indicating correlation with intelligence, especially for employees with less job experience $(-0,39 ; \mathrm{p}<0,05)$. The implications of these results for both research and practice are discussed.

Keywords: Personnel selection; validity; intelligence; electricity sector.
\end{abstract}

O surgimento da disciplina Psicologia Industrial ou Organizacional é quase tão antigo quanto o surgimento da própria psicologia (Landy, 1997). O uso de testes psicológicos nos processos de seleção de pessoal também é uma prática antiga dos psicólogos, datando do início do séc. XX (Ghiselli, 1973; Sampaio, 1998).

Os testes psicológicos, considerados procedimentos padronizados de discriminação de características psicológicas (Anastasi \& Urbina, 2000), começaram a ser utilizados em organizações a partir da construção de escalas de inteligência por Alfred Binet. Após Binet, na segunda década do século XX, os testes conhecidos como "Army Alfa" e "Army Beta" foram aplicados a recrutas do exército americano com fins de indicar características individuais de cerca de um milhão e meio de sujeitos (Sampaio, 1998).
Para uma inserção mais eficaz no contexto organizacional era necessário, no entanto, que os testes psicológicos se mostrassem eficientes na discriminação de critérios relevantes para o bom desempenho do funcionário na função. Nesse sentido, medidas como produtividade, número de acidentes de trabalho, avaliação de desempenho, entre outras, começaram a ser utilizados pelos psicólogos na tentativa de validar o processo de seleção de pessoal nas empresas (Sampaio, 1998).

Validade refere-se àquilo que um teste mede e ao quão bem ele faz isso (Anastasi \& Urbina, 2000). Segundo Pasquali (2001) um dos tipos de validade é a de critério, referindo-se ao grau de eficácia de um teste em predizer o desempenho relevante de um sujeito. Dependendo do espaço de tempo em que ocorre a coleta da informação pelo teste e a coleta da informação do critério, este tipo de evidência de validade costuma ser distinguido em validade preditiva ou concorrente (Pasquali, 2001).

Os Principles for the Validation and Use of Personnel Selection Procedures (Society for Industrial and Organizational Psychology, 2003), são um documento elaborado nos Estados Unidos com o objetivo de orientar os pesquisadores 
que trabalham com processos de validação de testes em empresas. De acordo com seus autores, o princípio essencial de qualquer processo de seleção de pessoal é a busca de evidências que ofereçam suporte às inferências feitas a partir dos escores obtidos pela avaliação psicológica e de sua relação com aspectos referentes ao desempenho no trabalho. A inferência básica é a de que um escore provindo de um determinado procedimento de seleção será capaz de predizer um subseqüente desempenho no trabalho. Também de acordo com os Principles, os procedimento usados em qualquer seleção devem apresentar suporte em evidências de validade.

Nos Estados Unidos a validação local para o uso de testes psicológicos passou a ser exigida nas empresas a partir da revisão do Ato de Direitos Civis de 1972 e das sucessivas regulamentações sobre o uso dos testes feitas pela Comissão de Iguais Oportunidades de Emprego (EEOC) e pelo Departamento de Trabalho do país (Resnick, 1982). A intervenção ocorreu nessa área, pois o governo se preocupava com a possibilidade de testes psicológicos discriminarem minorias e mulheres nos processos seletivos. A partir de então passou a ser exigido por lei que o empregador demonstre a relevância do teste em relação à perfor-mance do funcionário no trabalho. Além disso, a validade do teste deverá ser comprovada para o exercício de cada função na empresa. Nesse sentido, "as empresas foram proibidas de utilizar testes cujos estudos de validação tivessem sido feitos em outro lugar, mesmo que esses estudos fossem extensa e tecnicamente bem fundamentados" (Resnick, 1982, p. 78).

Os critérios de comprovação da relação teste-desempenho, validação local e exigência de comprovação de medidas não discriminatórias fizeram da testagem nas empresas nor te americanas um processo tão caro, que só as grandes companhias puderam custeá-lo. No entanto, uma adequação dos instrumentos à realidade da população avaliada passou a ser compromisso das empresas que lidam com processos seletivos.

No Brasil, medidas relacionadas à regulamentação do uso de testes são relativamente recentes. O Conselho Federal de Psicologia ([CFP], 2003) publicou uma Resolução (002/2003) que trata da regulamentação do uso, da elaboração e da comercialização dos instrumentos psicológicos no país. Esta Resolução prevê que os testes psicológicos sejam analisados por uma comissão consultiva com o objetivo de que apenas aqueles que apresentem os requisitos mínimos técnicos e científicos exigidos estejam disponíveis para uso pelos profissionais. As evidências de validade, entre outros, estão entre os requisitos psicométricos considerados, indicando a necessidade de adaptação à população avaliada para que representem de maneira mais acurada e fidedigna os resultados dos sujeitos submetidos ao processo de avaliação psicológica. De acordo com o Artigo 11 desta mesma Resolução: "As condições de uso dos instrumentos devem ser consideradas apenas para os contextos e propósitos para os quais os estudos empíricos indicaram resultados favoráveis" (CFP, 2003, p. 5). Este artigo reforça a importância de realiza- ção de pesquisas, inclusive as de validade, que evidenciem a eficácia de utilização dos testes para contextos particulares, como seria caso, inclusive, da área organizacional.

Schmidt e Hunter (1998), utilizando estudos de meta análise, investigaram e sumarizaram os resultados de 85 anos de pesquisa em seleção de pessoal, no que se referia à validade das medidas utilizadas. Examinaram os coeficientes de validade de 19 medidas destacando-se, dentre elas, testes de inteligência geral, provas situacionais, entrevistas, testes de integridade, testes de conscienciosidade, tempo de experiência no trabalho, testes de grafologia, nível de escolaridade, motivação para o trabalho e idade dos sujeitos. Como medidas de critério foram utilizadas a avaliação de desempenho e o nível de produtividade dos funcionários. Nos resultados, encontrou-se como medidas mais válidas as provas situacionais $(0,54)$, os testes que avaliam inteligência geral $(0,51)$ e a entrevista estruturada $(0,51)$. Os testes que avaliam personalidade, ou seja, os de integridade e os de conscienciosidade, apresentaram coeficiente de 0,41 e 0,31, respectivamente. A experiência no trabalho e os inventários de interesses apresentaram índices de 0,18 e 0,10. A grafologia e a idade dos sujeitos foram consideradas as medidas menos válidas, com coeficientes de 0,02 e - 0,01, respectivamente.

Em 1997, Herencia et al. realizaram em Brasília uma pesquisa com o objetivo de fazer um levantamento das técnicas e instrumentos utilizados por psicólogos e verificar a validade que lhes era atribuída pelos profissionais da área. Os sujeitos responderam a um questionário no qual deveriam listar as técnicas e instrumentos utilizados nas organizações e atribuir, a cada um deles, um grau de validade. Os resultados apontaram, entre outros, que a entrevista foi considerada a técnica mais válida e os testes psicológicos foram considerados pelos profissionais como os menos válidos, apesar de serem os mais utilizados, devido a uma praticidade de manuseio da técnica.

Em 2003 Pereira, Primi e Cobêro realizaram um estudo em 33 empresas do estado de São Paulo com o objetivo de identificar os testes utilizados em seleção de pessoal e verificar se os profissionais possuíam informações sobre a validade destes procedimentos. Os resultados revelaram alta utilização do teste Wartegg (15,2\%), dinâmicas de grupo $(15,2 \%)$ e de entrevista estruturada $(11,4 \%)$ e não estruturada $(7,6 \%)$ como métodos de seleção mais utilizados. A grafologia também aparece como uma técnica bastante usada, apresentado uma porcentagem de 6,8\% dos casos. Em relação à validade dos instrumentos, $85 \%$ dos entrevistados afirmam conhecer a validade do teste Wartegg e $88,9 \%$ a validade da grafologia. Em relação à escolha dos instrumentos por sua validade, $77,8 \%$ dos profissionais utilizam a grafologia porque a consideram uma técnica válida. Tal fato revela uma disparidade no fato de como os recrutadores compreendem o conceito de validade, já que muitos afirmam utilizar os instrumentos por sua validade, mesmo não existindo estudos empíricos sobre este parâmetro nos testes citados, como, por exemplo, o teste de Wartegg e a grafologia, sendo que, no caso desta última, o coefi- 
ciente de validade encontrado no estudo de Schmidt e Hunter (1998) foi de 0,02, ou seja, uma valor muito próximo a zero. Tal resultado aponta tanto para a necessidade de atualização dos profissionais que lidam com avaliação psicológica no contexto organizacional em relação à cientificidade dos instrumentos utilizados, quanto para a realização de pesquisas de evidências de validade de testes para os propósitos específicos da área organizacional, visto a carência de instrumentos validados para a área.

Os estudos citados anteriormente corroboram a utilização freqüente de testes psicológicos nos processos de seleção de pessoal em empresas. No Brasil, no entanto, essa utilização muitas vezes não é acompanhada de estudos que evidenciam a relação teste-critério para os cargos exercidos (Wanderley, 1985). Em relação aos estudos brasileiros estes se referem somente à padronização dos instrumentos utilizados e não buscam verificar as evidências de validade dos mesmos no contexto organizacional.

A Bateria de Provas de Raciocínio (BPR-5) é um instrumento utilizado para avaliação da inteligência geral e aptidões. Tal teste teve sua amostra de padronização composta por sujeitos de 11 a 18 anos, que cursavam de sexta a oitava série do ensino fundamental para a Forma A e de primeira a terceira série do Ensino Médio para a Forma B. Ainda não há relatos de estudos que tenham sido realizados com o instrumento para verificar se sua aplicação seria válida também para casos de seleção de pessoal. Neste sentido, o objetivo geral da presente pesquisa foi, então, verificar se haveria correlação entre o desempenho de funcionários submetidos à aplicação do BPR-5 com o número de acidentes de trabalho ocorridos com os mesmos.

\section{Método}

\section{Participantes}

Participaram do estudo 79 sujeitos, funcionários de uma empresa de energia elétrica brasileira. Todos exerciam, predominantemente, atividades com linha energizada, ou seja, trabalhavam diariamente em redes elétricas, sujeitos a uma carga elétrica de 13800 Volts, constituindo, assim, uma função de alto risco.

Os sujeitos da amostra foram predominantemente do sexo masculino e possuíam idade variando entre 19 e 45 anos. Estavam divididos em 53 eletricistas, 20 encarregados e 6 técnicos (supervisores). Possuíam escolaridade variando entre segundo grau incompleto e terceiro grau completo. O tempo de exercício na função variou de um a 24 anos.

\section{Instrumento}

BPR-5 - Bateria de Provas de Raciocínio

O BPR-5 (Almeida \& Primi, 1998; Primi \& Almeida, 2000a, 200ob) é um instrumento de avaliação das habilidades cognitivas que oferece estimativas do funcionamento cognitivo geral e das forças e fraquezas em cinco áreas específicas: (a) Raciocínio Verbal (RV), indicando extensão do vocabulário e capacidade de estabelecer relações abstratas entre conceitos verbais; (b) Raciocínio Abstrato (RA), indicando a capacidade de estabelecer relações abstratas em situações novas para as quais se possui pouco conhecimento previamente aprendido; (c) Raciocínio Mecânico (RM), avaliando o conhecimento prático de mecânica e física; (d) Raciocínio espacial (RE), indicando a capacidade em formar representações mentais e manipulá-las, transformando-as em novas representações e (e) Raciocínio Numérico (RN), indicando a capacidade de raciocínio com símbolos numéricos em problemas quantitativos e conhecimento de operações aritméticas básicas.

Para pontuação dos raciocínios, os escores brutos, constituídos pela soma dos acertos em cada subteste, são convertidos em EPNs (Escore-Padrão Normalizados), com média 100 e desvio padrão 15. Também é convertido em EPN o total de acertos em todos os subtestes da bateria. Além da pontuação em EPN, o manual do teste também fornece os valores em percentis para que seja possível a comparação de acertos dos sujeitos em relação ao grupo original de padronização da bateria, tanto para os cinco subtestes, quanto para o escore do total de acertos.

\section{Procedimentos}

A bateria de testes BPR-5 foi aplicada coletivamente, em dois dias de aplicação, sendo que no primeiro dia foram aplicados três subtestes do BPR-5, a saber, Raciocínios Verbal (RV), Abstrato (RA) e Mecânico e no segundo dia foram aplicados os subtestes Raciocínio Espacial (RE) e Numérico (RN).

Para mensuração dos acidentes de trabalho foi realizada uma consulta às Comunicações de Acidentes de Trabalho (CATs) disponíveis na empresa. Foram considerados somente os acidentes em que tenha sido comprovada responsabilidade do próprio funcionário em sua ocorrência. O acesso às CATs envolveu dados dos anos de 1998 até 2003. Para mensuração dos resultados tais dados foram divididos em acidentes ocorridos nos últimos cinco anos e nos últimos dois anos (dados mais recentes). No entanto, no momento de mensuração dos dados observou-se que não houve diferença estatisticamente significativa entre os acidentes sofridos nos últimos cinco e dois anos. Optou-se, portanto, por trabalhar com os dados de 2001 a 2003, envolvendo os acidentes sofridos nos últimos dois anos.

\section{Análise de Dados}

Foram calculadas estatísticas descritivas para caracterizar as variáveis e realizadas análises correlacionais para verificar as evidências de validade. Os cálculos foram realizados utilizando-se o programa estatístico SPSS, versão 2002.

\section{Resultados}

As estatísticas descritivas foram calculadas tanto para os testes quanto para os critérios. Os resultados serão apresentados a seguir. 
Baumgartl, V. O., \& Primi, R. (2006). Evidências de Validade da Bateria de Provas de Raciocínio (BPR-5) para Seleção de Pessoal.

Tabela 1

Estatísticas Descritivas do BPR-5 para o Escore Padrão Normalizado (EPN)

\begin{tabular}{llcccccc}
\hline BPR-5 & N & Mínimo & Máximo & Média & DP & Assimetria & Curtose \\
\hline RA & 79 & 67 & 129 & 94 & 11,7 & 0,546 & 0,518 \\
RV & 79 & 74 & 132 & 102 & 14,2 & 0,157 & $-0,758$ \\
RM & 79 & 76 & 146 & 111 & 12,4 & 0,300 & 0,665 \\
RE & 79 & 63 & 146 & 99 & 14,1 & 0,777 & 1,765 \\
RN & 79 & 75 & 146 & 103 & 15 & 0,778 & 0,891 \\
EG5 & 79 & 73 & 140 & 101 & 13 & 0,223 & 0,352 \\
\hline
\end{tabular}

Notas. Legenda. RA: Raciocínio Abstrato; RV: Raciocínio Verbal; RM: Raciocínio Mecânico; RE: Raciocínio Espacial; RN: Raciocínio Numérico; EG5: Escore total nas 5 provas.

De acordo com a Tabela 1 todos os sujeitos da amostra foram submetidos à aplicação da bateria e apresentaram maior média de desempenho nos Raciocínios Mecânico e Numérico. A média de todas as provas, representada pelo EPN total, encontra-se dentro da Zona Média de acordo com a classificação do Manual da bateria com o desvio padrão também dentro do esperado. A assimetria e a curtose foram positivas para a maioria das provas, revelando que a maioria dos sujeitos tendeu a apresentar um desempenho abaixo da média e a distribuição tendeu a ser leptocúrtica, com maior concentração ao redor da média.

Em relação aos acidentes de trabalho, as estatísticas descritivas foram calculadas para os acidentes ocorridos nos últimos dois anos. Os resultados podem ser vistos a seguir.

Tabela 2

Estatísticas Descritivas para o Número de Acidentes de Trabalho Ocorridos entre 2001 a 2003

\begin{tabular}{llll}
\hline $\mathrm{N}$ & $\mathrm{f}$ & $\%$ & $\mathrm{Fa}$ \\
\hline $\mathrm{O}$ & 73 & $\begin{array}{l}92,4 \\
7,6\end{array}$ & $\begin{array}{l}92,4 \\
100\end{array}$ \\
\hline & 6 & 100 & \\
\hline Total & 79 & & \\
\hline
\end{tabular}

Nota. Legenda. Fa: Freqüência acumulada.

Pela Tabela 2 pode-se visualizar que nos últimos dois anos o número máximo de acidentes sofridos foi um, o que representa uma porcentagem de 7,6\% de acidentes. 92,4 \% da amostra não sofreu nenhum acidente nos últimos dois anos. Passar-se-á agora aos resultados das correlações entre o desempenho nos subtestes e no critério.

Levando-se em consideração o fato de que o tempo de exercício na função pode influenciar o conhecimento da tarefa e, conseqüentemente, o desempenho no critério utilizado nesta pesquisa, foi realizado o cálculo das correlações testes-critério considerando o tempo de exercício da função, em número de anos. Como os participantes da pesquisa variavam de um a 24 anos de trabalho na linha viva, foi adotado o critério de separação em dois grupos, sendo que o primeiro possuía tempo de experiência de um a cinco anos e o segundo grupo de seis a 24 anos. Tal divisão foi realizada com base no artigo de Schmidt e Hunter (1998) no qual os autores realizaram um estudo similar com o objetivo de verificar o impacto do conhecimento da tarefa na performance do funcionário no trabalho. Os resultados encontram-se a seguir.

Tabela 3

Correlações entre o Desempenho no BPR-5 e o Número de Acidentes de Trabalho

\begin{tabular}{ll|l|l}
\hline BPR-5 & Total geral & $\begin{array}{c}\text { Acidentes } \\
\text { Grupo 1 }\end{array}$ & $\begin{array}{c}\text { Acidentes } \\
\text { Grupo 2 }\end{array}$ \\
\hline EG5 & $-0,072$ & $-0,390^{*}$ & 0,187 \\
RA & $-0,065$ & $-0,398^{*}$ & 0,144 \\
RV & $-0,241^{*}$ & $-0,371^{*}$ & 0,059 \\
RM & $-0,077$ & $-0,297$ & 0,109 \\
RE & $-0,161$ & $-0,421^{*}$ & 0,087 \\
RN & $-0,068$ & $-0,123$ & $-0,013$ \\
\hline
\end{tabular}

Nota.* $\mathrm{p}<0,05$.

Pela Tabela 3 pode-se observar correlações negativas e significativas para três provas do BPR-5 (RA, RV e RE) e para o escore geral na bateria (EG5) para o Grupo 01. Tal fato revela que as pessoas com menos tempo de experiência na função sofrem menos acidentes de trabalho à medida que revelam maiores notas em testes que avaliam a inteligência. Para o total das pessoas somente a prova RV apresentou correlação significativa, revelando que quanto maior a capacidade de raciocínio verbal dos funcionários, independente do tempo de experiência na função, menor a chance de ocorrerem acidentes de trabalho.

\section{Discussão}

Wanderley (1985) já afirmava que a avaliação psicológica no contexto or ganizacional deve estar firmemente amparada em estudos que não deixem dúvidas entre o que é medido pelo teste e o que é realizado no trabalho. Neste sentido, o presente estudo utilizou os testes referidos an- 
teriormente como preditorese como critério o número de acidentes sofridos no trabalho.

Em relação a este critério houve correlação negativa e significativa para os sujeitos com menor tempo de experiência na função, em relação ao desempenho nos subtestes Raciocínio Abstrato (RA), Raciocínio Verbal (RV), Raciocínio Espacial (RE) e para o escore total nas cinco provas (EG5). Tais subtestes avaliam principalmente inteligência fluida, referente à capacidade de raciocínio em situações novas, dependentes minimamente de conhecimentos adquiridos e inteligência cristalizada, referente à extensão e profundidade de conhecimentos adquiridos de uma cultura e à aplicação efetiva deste conhecimento (McGrew \& Flanagan, 1998).

Os resultados revelam que para pessoas com menor tempo de experiência numa função, o fator g (inteligência geral) desempenha um papel importante na aprendizagem dessas tarefas novas. À medida em que o funcionário apresenta maior conhecimento da tarefa, o valor preditor do fator g é menor. Tal afirmação já havia sido feita por Caretta e Doub (1998) e também é corroborada na presente pesquisa, uma vez que para os funcionários com maior tempo de experiência não houve correlações significativas com o BPR-5.

O Raciocínio Verbal (RV), referente à extensão do vocabulário e capacidade de estabelecer relações abstratas entre conceitos verbais, foi a única prova do BPR-5 que se correlacionou de maneira negativa e significativa com o total geral de acidentes, indicando que, independentemente do tempo de experiência na função, quanto melhor é o desempenho nesta prova, menor é a chance de um funcionário sofrer acidentes no trabalho. Tal fato pode ser explicado por uma particularidade do tipo de serviço que é realizado pela amostra do presente estudo. Toda vez, antes de executar um serviço, eles chegam ao local de trabalho e discutem passo a passo a resolução da tarefa, depois sobem na rede energizada, sujeitos à voltagem de 13.800 Volts, como referido anteriormente. Esta seria uma possibilidade de explicação do por que pessoas com maior capacidade de RV sofrem menos acidentes. Em relação aos valores das correlações encontrados, estes corroboram os achados da literatura de que os testes de inteligência são um bom preditor do desempenho no trabalho (Ghiselli, 1973; Kuncel, Hezlett \& Ones, 2004; Schmidt \& Hunter, 1998).

Apesar de ainda não haverem normas para interpretação dos escores do BPR-5 para a população adulta, a presente pesquisa teve como objetivo revelar os resultados de um estudo de validação da bateria. Os estudos de validação consistem em pesquisas que procuram evidências empíricas favoráveis às interpretações atribuídas aos escores do instrumento, em contextos específicos (American Educational Research Association, American Psychological Association and National Council for Measurement in Education,1999). Os estudos de normatização, os quais geralmente são feitos depois de já existir um amplo conjunto de evidências de validade, procuram estabelecer os parâmetros populacionais que indiquem as expectativas normais e os desvios, para que seja possível interpretar casos individuais (posicionando-os frente ao grupo de referência e indicando se determinado escore está abaixo, na média ou acima dela). É importante, então, ressaltar que a inexistência de estudos normativos impede a interpretação adequada de escores individuais, mas não impede que se possam efetuar estudos correlacionais entre certos índices obtidos pelo instrumento e outras variáveis, como se pretendeu fazer neste estudo.

\section{Conclusão}

Uma questão muito discutida hoje em dia pelos psicólogos organizacionais, principalmente os que trabalham com processos de seleção de pessoal, é: "o que realmente devese observar nos candidatos que se submetem a processos seletivos? Quais características eles devem possuir para serem bons funcionários no ambiente de trabalho?"

A presente pesquisa, em certa medida, tentou responder a esta questão. Observa-se que atualmente no Brasil os processos de seleção que utilizam testes psicológicos se baseiam quase exclusivamente em testes de personalidade, sendo que o contexto nacional ainda carece de pesquisas que investiguem o poder preditor de traços de personalidade em relação ao desempenho no ambiente de trabalho. A literatura internacional, no entanto, desde a década de 50 até os dias atuais, ressalta a importância dos testes de inteligência e da sua relação com a aprendizagem de tarefas. Os testes de personalidade também se revelam importantes, mas em menor grau.

Neste sentido a presente pesquisa reforça a necessidade da realização de estudos de validade com testes no Brasil, pois muitas características reveladas por um teste e que são o motivo de reprovação de candidatos, podem não ser características relevantes para um bom desempenho na função. Neste sentido, não adianta somente aprovar ou reprovar um candidato porque ele tirou uma nota baixa ou alta num teste, mas provar, de maneira científica, que aquela nota discrimina uma característica de um funcionário que desempenhará de forma segura e produtiva suas tarefas no ambiente de trabalho. É este o grande desafio dos psicólogos que lidam com seleção de pessoal hoje, no Brasil.

\section{Referências}

Almeida, L. S., \& Primi, R. (1998). Baterias de Prova de Raciocínio-BPR-5. São Paulo, SP: Casa do Psicólogo.

American Educational Research Association, American Psychological Association \& National Council for Measurement in Education. (1999). Standards for educational and psychological testing. Washington, DC: American Educational Research Association.

Anastasi, A., \& Urbina, S. (2000). Testagem psicológica (7. ed.). Porto Alegre, RS: Artes Médicas.

Caretta, T. R., \& Doub, T. W. (1998). Group differences in the role of $\mathrm{g}$ and prior job knowledge in the acquisition of subsequent job knowledge. Personnel Individual Differences, 23, 585-593. 
Conselho Federal de Psicologia. (2003). Resolução CFP $N^{\circ}$ Oo2/ 2003. Retirado em http://www.pol.org.br

Ghiselli, E. E. (1973). The validity of aptitude tests in personnel selection. Personnel Psychology, 26, 461-477.

Herencia, C. C., Moraes, M. Z., Nogueira, J. G. A, Rocha, F. C. A., Sombra, J. L., \& Torres, C. V. (1997). Instrumentos utilizados em seleção de pessoal: Sua validade aparente. Arquivos Brasileiros de Psicologia, 1(49), 31-42.

Kuncel, N. R., Hezlett, S. A., \& Ones, D. S. (2004). Academic Performance, career potential, creativity and job performance: Can one construct predict them all? Journal of Personality and Social Psychology, 86(1), $148-161$.

Landy, F. J. (1997). Early influences on the development of industrial and organizational psychology. Journal of Applied Psychology, 82(4), 467-477.

McGrew, K. S., \& Flanagan, D. P. (1998). The intelligence test desk reference (ITDR): $G f-G c$ cross-battery assessment. Needham Heiths, MA: Allyn \& Bacon.

Pasquali, L. (2001). Técnicas de exame psicológico - TEP I. São Paulo, SP: Casa do Psicólogo.

Pereira, F. M., Primi, R., \& Cobêro, C. (2003). Validade dos testes utilizados em seleção de pessoal segundo recrutadores. Psicologia: Teoria e Prática, 5(2), 83-98.

Primi, R., \& Almeida, L. S. (2000a). Estudo de validação da bateria de provas de raciocínio (BPR5). Psicologia: Teoria e Pesquisa, 16(2), 165-173.

Primi, R., \& Almeida, L. S. (2000b). BPR-5. Bateria de provas de raciocínio. Manual técnico. São Paulo, SP: Casa do Psicólogo.

Resnick, L. B. (1982). Test in America: The current challenge. International Review of Applied Psychology, 31(1), 76-90.

Sampaio, J. R. (1998). Testes psicológicos no processo de seleção de pessoal. In I. B. Goulart, \& J. R., Sampaio, Psicologia do trabalho e gestão de recursos humanos: Estudos contemporâneos (pp. 149-167). São Paulo, SP: Casa do Psicólogo.

Schmidt, F. L., \& Hunter, J. E. (1998). The validity and utility of selection methods in Personnel Psychology: Practical and theoretical implications of 85 years of research findings. Psychological Bulletin, 124(2), 262-274.

Society for Industrial and Organizational Psychology. (2003). Principles for the validation and use of personnel selection procedures. Retirado em http://www.siop.org

Wanderley, W. M. (1985). Os testes psicológicos em seleção de pessoal: Análise crítica dos conceitos e procedimentos utilizados. Arquivos Brasileiros, 2(37), 16-31. 